\title{
Global assessment of organ specific basal gene expression over a diurnal cycle with analyses of gene copies exhibiting cyclic expression patterns
}

Yuan Lu", Mikki Boswell', William Boswell', Raquel Ybanez Salinas ${ }^{1,2}$, Markita Savage $^{1}$, Jose Reyes ${ }^{1}$, Sean Walter ${ }^{1}$, Rebecca Marks ${ }^{1}$, Trevor Gonzalez ${ }^{1}$, Geraldo Medrano ${ }^{1}$, Wesley C. Warren ${ }^{3}$, Manfred Schartl ${ }^{1,4}$ and Ronald B. Walter ${ }^{1}$

\begin{abstract}
Background: Studying functional divergences between paralogs that originated from genome duplication is a significant topic in investigating molecular evolution. Genes that exhibit basal level cyclic expression patterns including circadian and light responsive genes are important physiological regulators. Temporal shifts in basal gene expression patterns are important factors to be considered when studying genetic functions. However, adequate efforts have not been applied to studying basal gene expression variation on a global scale to establish transcriptional activity baselines for each organ. Furthermore, the investigation of cyclic expression pattern comparisons between genome duplication created paralogs, and potential functional divergence between them has been neglected. To address these questions, we utilized a teleost fish species, Xiphophorus maculatus, and profiled gene expression within 9 organs at 3-h intervals throughout a 24-h diurnal period.
\end{abstract}

Results: Our results showed 1.3-21.9\% of genes in different organs exhibited cyclic expression patterns, with eye showing the highest fraction of cycling genes while gonads yielded the lowest. A majority of the duplicated gene pairs exhibited divergences in their basal level expression patterns wherein only one paralog exhibited an oscillating expression pattern, or both paralogs exhibit oscillating expression patterns, but each gene duplicate showed a different peak expression time, and/or in different organs.

Conclusions: These observations suggest cyclic genes experienced significant sub-, neo-, or non-functionalization following the teleost genome duplication event. In addition, we developed a customized, web-accessible, gene expression browser to facilitate data mining and data visualization for the scientific community.

Keywords: Xiphophorus, Organ, Gene expression profiling, Profiling, Light response, Basal level gene expression, Paralog, Genome duplication, Evolution

\footnotetext{
* Correspondence: y_154@txstate.edu

${ }^{1}$ The Xiphophorus Genetic Stock Center, Department of Chemistry and Biochemistry, Texas State University, 419 Centennial Hall, 601 University Drive, San Marcos, TX 78666, USA

Full list of author information is available at the end of the article
} 


\section{Background}

There are over 33,000 described fish species, comprising the largest vertebrate group. Teleost fishes make up $96 \%$ of all extant fish species and exhibit extreme biodiversity that is thought to be a result of the teleost specific whole genome duplication (TGD) event that is estimated to have occurred $\approx 375$ million years ago [1-4]. This duplication, accompanied with further retention of duplicated gene copies (i.e., neo-, sub-, non-functionalization, and chromosomal structural change), shaped the genomes of the teleost common ancestor and allowed each species to tailor its genome to best fit their particular environmental niche [5-8]. Studies of whole genome duplication (WGD) events have provided data to support the ability to track changes in gene function by comparing post-WGD duplicated allele pairs to pre-WGD single genes [9].

It is well known that gene expression does not remain at a steady state over a daily light/dark cycle but is dynamically responsive to a variety of environmental stimuli [10-12]. The dynamic nature of gene expression leads to transcriptional profiles in each organ that reflect complex time-of-day expression patterns that are tuned to properly regulate physiological demands. Genes known to follow cyclic ebbs and flows in their expression patterns serve as examples of the complex homeostatic genetic regulatory networks, yet these cycles only account for one aspect of many potentially differing oscillating gene expression patterns [13]. There are many other instances where basal gene expression at first appear to exhibit random patterns, yet later are shown to actually reflect a dynamic response to an extrinsic temporal stimuli, such as light-dark switching, feeding and other subtle adaptations to environmental conditions [14-19].

Circadian oscillation of gene expression has been shown for all vertebrate organisms thus far studied and is known to affect physiology in different model systems [20]. It is also well known that a group of genes (i.e., circadian genes) have expression patterns that coincide with discrete physiological changes in the diurnal cycle [21-23]. Even Mexican blind cavefish and Antarctic blackfin icefish encode circadian genes in their genomes $[24,25]$. Although the blind cavefish do not exhibit per1 circadian rhythm in the wild, it can be entrained in the lab with light. Although it is observed that different members of the same circadian gene family may exhibit different expression patterns, how gene duplicates, especially those originating from a WGD event (i.e., Ohnologs) behave transcriptionally has not yet been studied $[26,27]$. This critical piece of missing information may be useful to infer the molecular evolution of rhythmicity.

The resolution of duplicated genomes in teleost fishes is hypothesized to have taken place soon following the duplication (i.e., 70-80 million years following the duplication), and most genes resolved down to a single copy during that period. However, the genomes of different extant teleost species may contain $\approx 3-20 \%$ of their genes in a duplicated condition when compared to basal actinopterygians (i.e., fishes that did not experience TGD), or tetrapods $[8,9,28,29]$. Functional analyses showed a majority of the paralogs developed new functions compared to the molecular ancestor. We hypothesize that most paralogs of genes exhibiting cyclic patterns are differentiated into different expression patterns following genome duplication. In this report, we use cyclic expression patterns as a function to compare paralog functions.

It is possible to track WGD event and re-construct the ancestral teleost chromosomes that are hypothesized to share similar structures to those in tetrapods, including human $[9,28]$. It has also been shown that teleost genomes represent excellent models to study molecular evolution. We chose Xiphophorus as the model for this study for several reasons: 1 . X. maculatus is a new world, live-bearing vertebrate with an inbred (i.e., 115 generations of sibling matings) pedigree, 2. Xiphophorus fishes represent a well-established biomedical model for studies aimed at determining the genetics underlying melanoma development [30], 3. Xiphophorus genomes (i.e., $X$. maculatus, $X$. couchianus and $X$. hellerii) represent some of the best-assembled vertebrate genomes in existence, in terms of chromosome contiguity, sequence gaps and unassembled contigs [31, 32]. Also, availability of genomes from three distinct species within the same genus allows inter-species comparative genomic and transcriptomic studies to be performed [17, 33-35]; 4. Xiphophorus belongs to teleost fishes that have retained portions of their genomes in duplicated form, enabling the investigation of genomic molecular evolution [4, 29]; 5. A unique feature of Xiphophorus fishes is the ability to produce viable interspecies hybrids. This attribute presents researchers with a hybridization model that allows genetic interactions between divergent alleles that are artificially brought together in the interspecies hybrids to be studied [33-35].

Collectively, this study collects a comprehensive list of organ-specific genes exhibiting cyclic expression patterns (i.e., cyclic genes). In addition, comparing the paralog expression patterns forwards our understanding of the molecular evolution of these genes.

\section{Results}

\section{Gene expression profiling statistics}

Gene expression for 9 organs (brain, eye, gills, ovary, testis, heart, liver, muscle, and skin) was profiled every 3 $\mathrm{h}$ over a 24-h period from two fish at each time point using RNA-Seq. Overall, 87.9-92.2\% of 46.1-51.8 
Table 1 RNA-Seq statistics

\begin{tabular}{lllll}
\hline Organ & Average filtered reads $(\mathbf{M})$ & Average mapped reads $(\mathbf{M})$ & Average mapping rate $(\%)$ & Average depth $(\times)$ \\
\hline Brain & 46.1 & 41.9 & 90.8 & 98.2 \\
Eye & 51.8 & 46.5 & 89.8 & 110.3 \\
Ovary & 50.6 & 46.1 & 91.2 & 107.7 \\
Testis & 49.6 & 45.6 & 92.1 & 105.6 \\
Heart & 49.7 & 43.6 & 87.9 & 105.7 \\
Liver & 48.6 & 43.2 & 88.8 & 103.5 \\
Muscle & 51.5 & 45.2 & 87.9 & 109.6 \\
Skin & 46.8 & 42.4 & 90.5 & 99.7 \\
\hline
\end{tabular}

M Million

million (M) filtered sequencing reads were mapped to the $X$. maculatus reference genome v5.0, with an average sequencing depth within exonic regions of 98.2-110.3 $\times$ in different organs (Table 1). This dataset was further processed as library size-normalized read counts (i.e., cpm). Visualization and data mining of this dataset can be accessed by https://www.xiphophorus.txstate.edu/ Gene-Expression-Browser.html.

Expression patterns of circadian master regulator families The Xiphophorus genome encodes 3 arntl genes (one arntl1a, two arntl2), 2 clock genes, 4 per genes (one per $1 b$, one per 2 , one per 3 , and one per 2 paralog named period circadian regulator 2) and 7 cry genes [three cry1, one cry2, one cry-dash, cry5 (6,4 photolyase) and CPD photolyase zgc:66475] (Fig. 1). All, except for cry-dash and cry5, showed circadian expression patterns as expected. These findings serve as a positive control and validate cyclic gene discovery presented in this study: known circadian master regulator genes show cyclic expression patterns and peak expression times at similar zeitgeber time $(\mathrm{Zt})$ in all 9 organs although testis and ovary exhibited smaller amplitude for all circadian master regulator genes (Fig. 1). The cry-dash and cry5 genes do not show cyclic expression pattern, but exhibited peak expression in light-phase. The cry-dash gene exhibited statistically different expression between early light and late dark phase in skin, brain and eye; and cry5 in brain, eye, and muscle (Fig. 1d; Supplement Table 1). Their expression patterns suggest they are lightinducible genes (Fig. 1d).

\section{Identification of genes exhibiting cyclic expression} patterns in 9 Xiphophorus organs (skin, brain, liver, gills, testis, ovary, heart, eye and muscle)

Gene expression profiling was performed on 9 organs throughout a $24 \mathrm{~h}$ cycle, at a $3 \mathrm{~h}$ intervals. Genes exhibiting cyclic expression pattern were subsequently identified ( $p$-value $<0.01$; normalized gene expression value, cpm, is larger than 1 in at least two time points; skin: 935; brain: 731; ovary: 224; heart: 659; muscle: 2076; eye:
4033; gill: 1740; liver: 1039; testis: 294; Fig. 2; Supplement Tables 2 and 3). There are 34 opsin genes encoded in the $X$. maculatus genome. Except opn6, expressed in all tested organs, and universally unexpressed opn $7 a$, opn1sw1, tmtops3a and parietopsin, each organ exhibits a different opsin expression profile (Supplement Figure 5a). Although eye expressed most opsin genes (i.e., 28 of the 34) and showed the largest number of cyclic genes (Supplement Figure 5a; Supplement Table 2), the number of opsin genes expressed in any organ is not correlated to the number of cyclic genes. However, lws2, opn $6 b$, rrh, parapinoopsin and lws 4 are only expressed in eyes. Considering that cyclic genes are the most abundant in the eyes, it implies that these genes may be related to the higher ocular representation of cyclic genes.

Genes showing the cyclic expression patterns were mostly organ specific (Supplement Figure 5b). This remains true even among comparisons that exclude testis and ovary, that exhibited the fewest cyclic genes and therefore showed smallest overlap with the circadian gene sets of other organs.

\section{Identification of Ohnologs and singletons Xiphophorus compared to the spotted gar genome}

The $X$. maculatus genome (assembly version 5.0; Ensembl release v94) encodes a total of 24,209 genes. We have identified 1886 Xiphophorus genes that show 2: 1 relationship to gar (i.e., 943 Ohnolog pairs; Fig. 3; Supplement Table 4), and 10,952 X. maculatus genes that show 1:1 to gar (i.e., singletons; Supplement Table 5). Therefore, a total of 12,838 Xiphophorus genes (53\%) can be tracked through the TGD using gar genome as a bridge. For example, among the Xiphophorus arntl, cry, per and clock gene families, per 2 and period circadian regulator 2, crylab and crypthochrome-1-like, and two arntl2 genes are duplication products of unnamed gar genes ENSLOCG00000004441, crylab, and arntl2, respectively (Supplement Figure 6).

An average of $65.2 \%$ of the cyclic genes from the different organs can be traced to singletons or Ohnologs (Supplement Table 6). It is hypothesized herein that two 

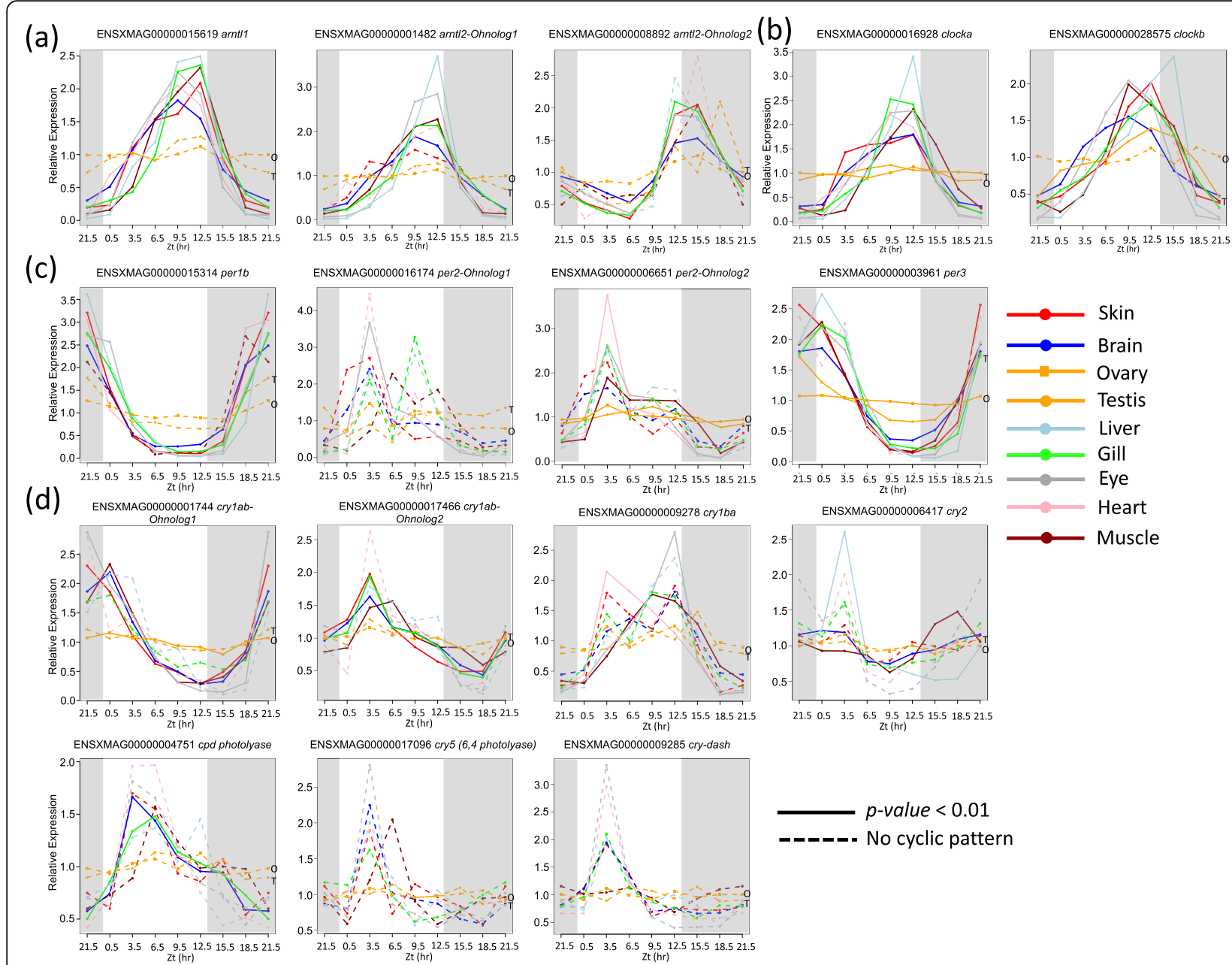

Fig. 1 Master circadian regulator gene expression throughout a diurnal cycle As a technical control, each member of known circadian master regulator gene families in the $X$. maculatus genome was tested for a cyclic gene expression pattern. These genes include a: The arntl gene family, $\mathbf{b}$ : The clock gene family, c: The per gene family, and $\mathbf{d}$ : The cry gene family. For each gene, relative expression values that were calculated by normalizing average absolute expression to daily expression average are plotted against zeitgeber times to from an organ-specific expression pattern. Expression patterns of 9 organs are plotted, with colors represented organ, and line types represented if a gene exhibit cyclic expression pattern. Gray and white areas represent dark and light phases respectively

Ohnologs preserve the ancestral function, meaning in most cases both Ohnologs should exhibit the same spatial and temporal cyclic based expression pattern. However, it was observed to be very rare for both Ohnologs to exhibit cyclic based expression patterns (i.e., average of only $1.6 \%$ of genes; Supplement Table 6) regardless of the expression pattern tested. Therefore, most post-TGD duplicates exhibit neo- (i.e., molecular ancestor exhibit no cyclic cycle, one or both Ohnologs exhibit cyclic cycle) or sub-functionalization (i.e., molecular ancestor exhibit cyclic expression, one Ohnolog lost it, or both Ohnologs exhibit cyclic cycles, but expression patterns do not overlap), instead of superfunctionalization (i.e., both Ohnologs showed the same cyclic expression pattern).

\section{Functional divergence of cyclic genes in Xiphophorus}

Although it is impossible to determine if a gene duplicate gained a new function, or lost an ancestral function after a WGD event without knowing the ancestral genetic function, it is important to know how Ohnologs differentiate in function (or not) to study how WGD and the following genome retention patterns have reshaped the cyclic rhythm regulatory circuits. Of the 964 Ohno$\log$ pairs that showed a 2:1 relationship between the $X$. maculatus and gar, 626 of them exhibit cyclic expression patterns for at least one Ohnolog in at least one organ (Supplement Figure 7). Also, 4573 singletons exhibit cyclic based expression patterns among the various different organs (Supplement Figure 8). A majority (i.e., 86.3$100 \%$ ) of cyclic Ohnologs exhibit intra-organ functional 


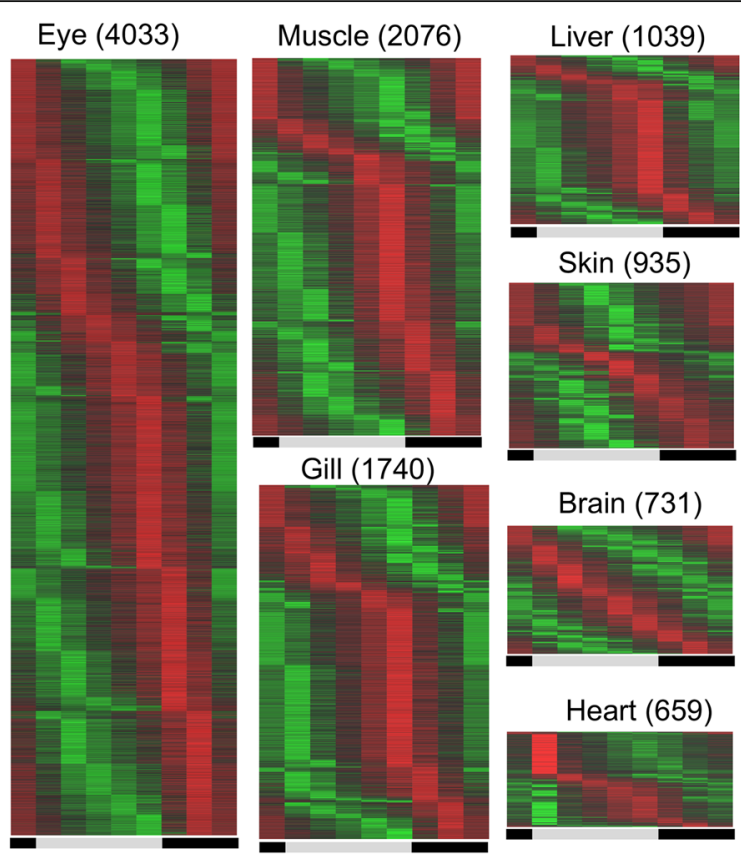

Fig. 2 Cyclic genes in each organ: Circadian genes within each organ were identified by rain algorithm ( $p$-value $<0.01)$. Each heatmap represents the circadian gene expression patterns of all genes in that organ over a 24-h period. Lists of circadian gene names and expression levels for each time point are provided in Supplemental Table 3). Gene expression read counts were first normalized to library size to generate CPM values. Mean of CPM values between biological replicates were subsequently calculated to represent expression level of a gene in an organ at a particular Zt, followed by scaling of gene expression at each Zt to the mean expression of a gene throughout a day. The scaled expression levels were represented by color (i.e., red: higher expression; green: lower expression). Circadian genes were ordered by peak expression time and length of time for a gene to reach the minimum expression

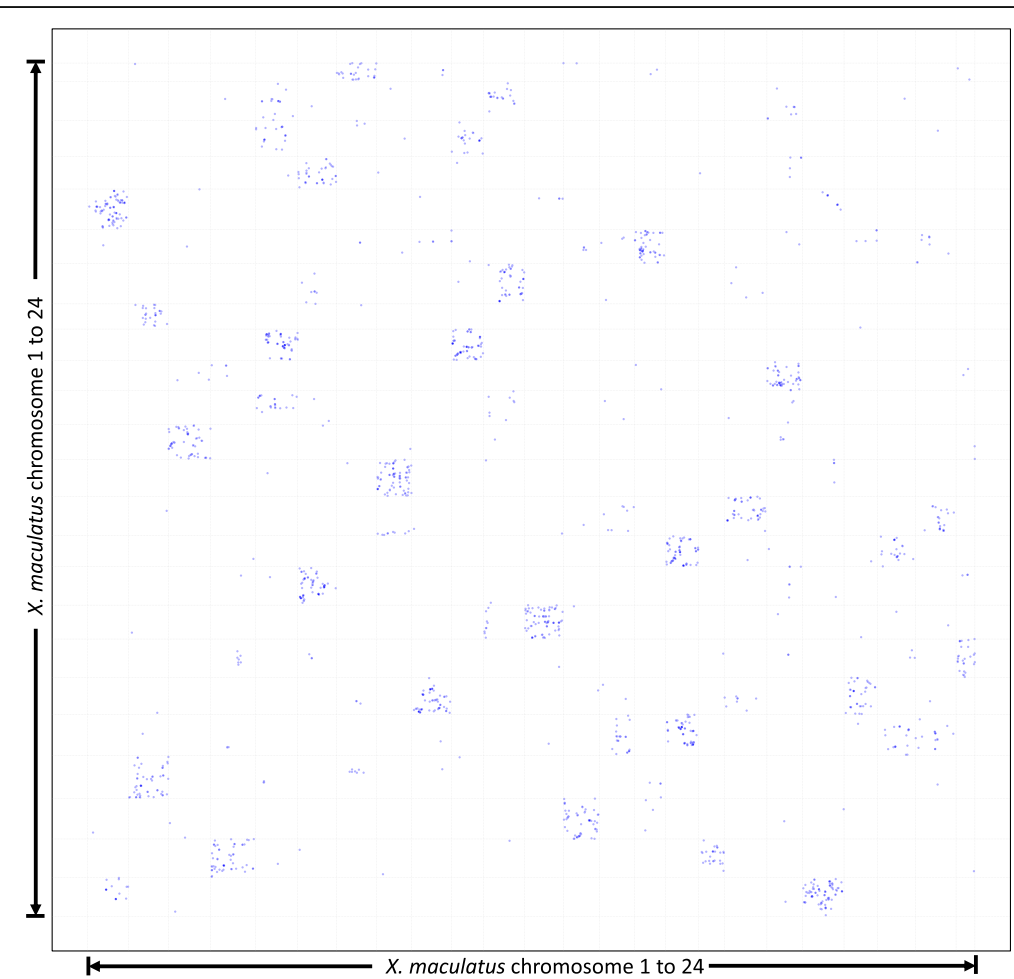

Fig. 3 Gene duplication in X. maculatus genome compared to gar Chromosome plots showing all genomic locations of 943 Ohnolog pairs. $X$ and $Y$ axis represent chromosome lengths. Each spot on the graph represents one Ohnolog pair that are both orthologs to a single gar gene. The $X$ - and $Y$-coordinates of each point correspond to the genomic location of each paralog gene on the Xiphophorus chromosome 
differentiation (i.e., only one of the pair exhibit cyclic gene expression per organ, or both Ohnologs exhibit cyclic expression patterns, but show cyclic pattern different from one another), and only a small fraction (i.e. 2.6\%) of these genes exhibit peak expression at the same time in the same organs (i.e., superfunctionalization; Supplement Table 7). Due to the high organ specificity of cyclic gene expression, all of the 626 Ohnolog pairs exhibited inter-organ functional differentiation (i.e., only one of the gene pairs exhibited cyclic gene expression in various organs, or both Ohnologs exhibit cyclic expression patterns, but show peak expression at different times among different organs; Supplement Table 7).

A survey conducted on zebrafish brain circadian genes (http://cgdb.biocuckoo.org/) showed similar observations, that a majority (29 of 32 Ohnolog pairs) of the genes do not share the expression pattern with their Ohnologs (Supplement Table 8). Also, 4 of the 7 genes that show a circadian expression pattern in both brain and pineal gland exhibited different peak expression $\mathrm{Ct}$ times (Supplement Table 9). These observations, from a teleost fish model distant from Xiphophorus, may suggest that circadian gene sub-functionalization following TGD is a feature shared among fish species.

\section{Intra-organ functional divergence}

Of the 626 Ohnolog pairs that exhibit circadian expression patterns for at least one of the duplicated genes, 340 pairs $(54.3 \%)$ are also organ specific. Among these are 295 cases where one gene exhibits a cyclic expression pattern, but not the other; 21 cases where both duplicated genes exhibit cyclic expression, but have different expression patterns; and 24 cases where both duplicated genes cycled with the same expression pattern (Fig. 4a; For Ohnologs expression pattern, see Supplement Figure 4).

\section{Inter-organ functional divergence}

286 of the 626 circadian Ohnolog pairs exhibited circadian expression patterns in multiple organs. These include 126 cases where only one Ohnolog exhibit circadian expression pattern; 159 cases where different Ohnolog exhibit cyclic expression pattern in different organs; and only one case where both Ohnolog show synchronized cyclic cycle in several organs (Fig. 4b; For Ohnologs expression pattern, see Supplement Figure 7). 1427 of the 4573 singleton cyclic genes are present in multiple organs, 423 genes exhibited different expression pattern in different organ and 1004 exhibited synchronized cyclic pattern in different organs (For Ohnologs expression pattern, see Supplement Figure 8).

Taken together, Ohnolog pairs mostly exhibited differentiated expression patterns throughout a 24-h period that contains both dark and light phases. The observed expression pattern differentiation suggested the fate of Ohnologs following WGD is predominantly subfunctionalization.

\section{Discussion}

Gene expression is a dynamic process and under the regulation of constantly changing environmental stimuli $[10,11]$. Therefore, researchers should take the complexity of basal gene expression into consideration when investigating biological effects when studying genetic functions for a few simple reasons: (a) Target gene(s) of interest need to be expressed in target organ(s) in order for functional tests, such as knock-down or knock-outs, to be interpreted; (b) Gene(s) that may functionally compensate a target of interest need to be taken into account (e.g., paralogs); (c) Since basal gene expression is often time-dependent and external stimulus related (e.g., circadian genes, light inducible genes), a proper time to apply an experimental intervention needs to be determined. In consideration of these experimental parameters, we assembled a basal gene expression browser to assist researchers in addressing the above concerns. Major genome databases, such as NCBI, ENCODE and Ensembl, carry organ specific gene expression data for a variety of species, but lack temporal expression pattern data along with it. The dataset and expression browser presented in this report therefore serves as a new and supplementary tool to those databases.

We detailed expression patterns of four circadian regulator gene families (i.e., ARNTL, CLOCK, PER and CRY), as examples and technical controls for further investigation, to demonstrate the power of our platform to detect and display gene expression patterns showing temporal shifts during the diurnal period. ARNTL and CLOCK genes form heterodimers to induce transcription of PER and CRY genes, therefore ARNTL and CLOCK expression patterns are expected to be synchronized and diurnally opposite to PER and CRY [For review, see [23]]. This is true for both of the $X$. maculatus clock genes, and two of the arntl genes (i.e., arntl1, and anrtl2-ohnolog1) although in some cases (e.g., heart, testis) a family member shows circadian patterns that are organ-specific. In contrast, the arntl2-Ohnolog 2, not only lost cyclic expression pattern in most of the assessed organs, but also shows peak expression that is shifted to the middle of dark phase, instead of at the light-dark phase transition period (Fig. 1). This observation suggests sub-functionalization following the TGD and warrants investigation into the function of the arntl2 duplicate. In contrast, PER and CRY genes show peak expression $\sim 6 \mathrm{~h}$ following ARNTL and CLOCK peak expression times, or at the dark-light transition. Both per1b and per3, as well as crylab Ohnolog 1, exhibit the anticipated expression patterns (Fig. 1). Both of 

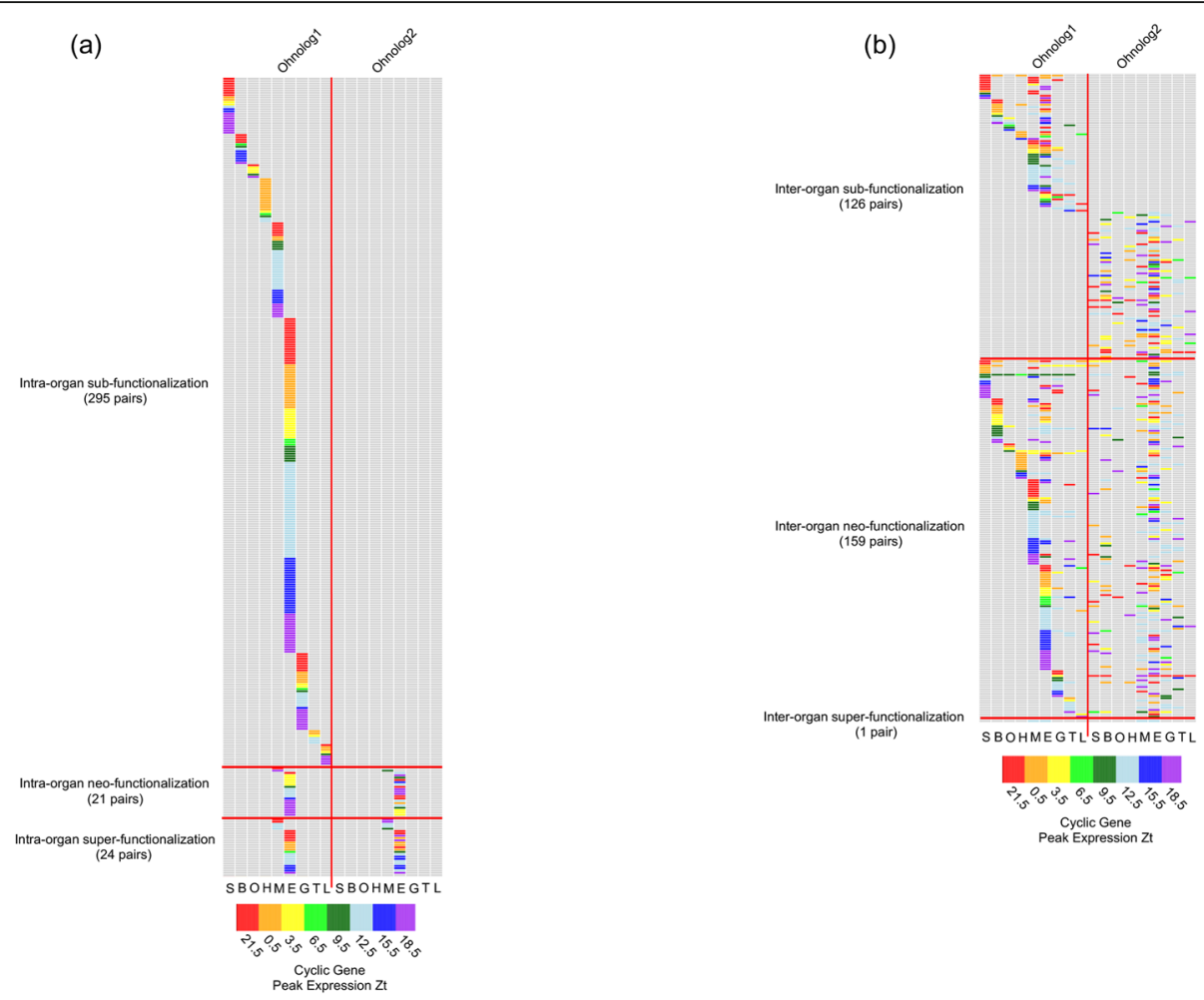

Fig. 4 Functional divergence of Ohnologs showing cyclic expression pattern: a intra-organ functional divergence, and $\mathbf{b}$ inter-organ functiona divergence. In each case, heatmap was used to describe the organ(s) in which gene showing circadian pattern. Colored blocks represent a particular gene that exhibits circadian expression in a particular organ, with the color of the block represented the peak expression Zt. Light gray means no circadian expression was identified. The center red line dividing each heatmap, splits the left and right half of a heatmap, each half represents Ohnolog1 and Ohnolog2, respectively. A majority of Ohnolog pairs showed Ohnolog-specific circadian expression patterns (i.e., suband neo-functionalization). S=Skin; B=Brain; $\mathrm{O}=$ Ovary; $\mathrm{H}=$ Heart; $\mathrm{M}=$ Muscle; $\mathrm{E}=\mathrm{Eye} ; \mathrm{G}=\mathrm{Gills}$; $\mathrm{L}=$ Liver; $\mathrm{T}=$ Testis

the per2 genes are Ohnologs. However, per2-Ohnolog1 lost circadian expression in all organs, while per2-Ohno$\log 2$ exhibited an unequally shifted expression pattern. This indicates the per 2 genes may have lost tight transcriptional regulation in the Xiphophorus lineage and therefore may not serve as sound markers for circadian studies. The cry gene family represents the most complex basal gene expression pattern of those discussed herein. The cry1ab-Ohnolog2, cry $1 b a$ and cry 2 all show shifted peak expression towards the light-dark transition, with the cry $1 b a$ expression pattern resembling that of arntl and clock, as observed in zebrafish [36]. Three fish specific cry genes, cpd photolyase, 6,4 photolyase (cry5) and cry-dash exhibit high expression only in light phase of a 24-h period (Fig. 1; Supplement Table 1). This observation corresponds to their presumed function in repair of UV induced DNA damage [37-39]. The same observation that cpd photolyase, 6,4 photolyase exhibited up-regulation following $2 \mathrm{~h}$ of FL light exposure was made in our previous study [40, 41]. The current study added more dynamic information to the gene expression change. The instantaneous increase of both genes' transcripts suggests the up-regulation may due to post-transcriptional modulation (e.g., decrease of transcript degradation), rather than enhancement of new transcript synthesis. The molecular evolution of these photolyases, as well as their functional divergence from visible light-inducible DNA repair genes for UV induced damage in fishes, instead of circadian regulators as in mammals, is an interesting research topic. The complex transcriptional behavior of this family of genes supports the importance of studying basal gene expression as a first step in genetic analyses of biomedical research topics.

We found that among all the organs tested eye exhibited the highest percentage of cyclic genes. 18,377 genes are expressed in eye, and $4033(21.9 \%)$ of them showed cyclic expression patterns (Fig. 2; Supplement Table 2 and 3). This is expected considering that eye is the major light-receiving organ, and cyclic gene expression may be light inducible. Although cycling expression patterns of opsins are not the prerequisite for cyclic rhythm, eyes did express most the Xiphophorus opsin genes and also exhibited the largest number of opsins exhibiting cyclic expression patterns (Supplement Figure 5). This suggests the cyclic pattern of a large portion of the ocular 
transcriptome is driven by opsins and their downstream signaling pathways. In contrast, ovary and testis exhibited the least amount of cyclic genes. Transcriptional rhythmicity studies performed in mammalian model systems have shown mixed results regarding gonad circadian gene expression (i.e., cyclic or non-cyclic) [42-48]. This may be due to the different types of molecules measured (i.e., RNA transcripts vs. protein) or sensitivity of the bioassays. We found known circadian regulators did show cyclic expression patterns in gonads, but the amplitudes are very small when compared to other organs, and so exhibited a relatively "muted" pattern of expression. These results support others that indicate gonads have cyclic transcriptional rhythmicity, and also expand potential circadian genes to non-master regulators within gonad, and therefore forward our understanding of transcriptional homeostasis in reproductive organs.

Genomic studies, accompanied with natural biodiversity, enable the study of molecular evolution by comparisons, and translation of discoveries from various aquatic animal models into human biomedical applications [30]. TGD provides the raw genetic material for the formation of the clade comprising the largest numbers of species and also provides a plethora of information for study of molecular evolution and genetic functional adaptation. However, the TGD genome duplication event also confounds identification of orthology between pre- and post-TGD species (e.g., teleost and tetrapods). The recent sequencing of gar genome facilitated amelioration of this confusion because gar has maintained a fish-like lifestyle but did not undergo the teleost genome duplication, and therefore serves as a direct connection between teleost fish and mammals $[49,50]$. We were able to trace 12,838 Xiphophorus genes to 11,895 gar genes $(10,952$ singletons and 943 ohnolog pairs; Fig. 3; Supplement Tables 4 and 5). These numbers are similar to what were reported in comparative genomic studies performed between medaka and zebrafish [9]. Because it is impossible to assess gene expression patterns of the common ancestor between Xiphophorus and gar, and similar gar basal gene expression data over time is not readily available, we cannot truly access the functional divergence between Xiphophorus Ohnologs. However, characterizing how the gene duplicates behave differently is significant to study molecular evolution of particular gene families, and within the scope of the presented study, the fate of cyclic genes following the TGD event. Therefore, we investigated cases where only one gene of the Ohnolog pair exhibits cyclic gene expression, but not the other, or two Ohnologs exhibit different expression patterns within the same organ or among different organs (i.e., subfunctionalization), and both Ohnologs exhibit cyclic expression patterns, but show different cyclic expression peaks (neo-functionalization), under the assumption that molecular ancestors of the Xiphophorus cyclic genes are also cyclic. It is intriguing that Ohnologs exhibiting suband neo- functionalization account for a majority of the genes showing cyclic expression patterns. This observation suggests that most Ohnologs within a duplication pair have evolved new functions and therefore are not likely to be functionally and temporally redundant to their partners. This raises the question of why only one Ohnolog gene is selected to carry the cycling expression pattern (Fig. 4; Supplement Table 7), or conversely, what are the effects of Ohnolog overexpression. It was suggested that complex genes are preferentially retained as duplicates following TGD [51]. Therefore, we investigated complexity of gene structure (i.e., gene, transcript, CDS, UTR lengths) between the cyclic and non-cyclic gene copies, to test if differential complexity of Ohnologs might differentiate their expression patterns. However, we found no association between these parameters and both expression patterns. Additionally, expressivity of Ohnologs, and the relative expression levels between two Ohnologs in a pair did not correlate to cyclic expression patterns (Supplement Figure 9). Therefore, the sub-functionalization in circadian expression patterns may be rooted in more complex divergence mechanisms within select regulatory regions, or molecular interactions within the context of the whole transcriptome, epigenetic regulation, or DNA/RNA sequences throughout post-TGD evolution.

Master circadian regulator genes were found in a few fish species and their expression patterns appear to be consistent to their mammalian counterparts [52, 53]. Following the teleost genome duplication, most duplicated genes were resolved back to one copy (i.e., singleton) while only $\approx 5-20 \%$ of the ancestral teleost genome remained duplicated $[8,9,54]$. It is important to know whether and how the Ohnologs may differ in terms of cyclic expression pattern, in order to study the molecular evolution of these genes, and deconvolute regulatory mechanisms underlying these expression patterns. Although comparative genomics for known circadian regulators (e.g., per, cry, arntl, and clock genes) have been performed for a few fish species [26], functional comparison of such genes is not studied at transcriptome level. We extended the inter-Ohnologs expression patterns comparison to zebrafish by incorporating reported zebrafish circadian genes in brain and pineal gland, and identified Ohnolog pairs $[9,55]$. It is showed that a majority Ohnolog genes do not shared cyclic expression patterns (Supplement Tables 8 and 9). These observations are in accordance to Xiphophorus and suggest that cyclic gene sub-functionalization following TGD is a common fate of retained gene copies. Similar observations that sub- and neo-functionalization account for a majority of 
the extant Ohnologs functions are not unprecedented. Pasquier et al. reported a majority of Ohnologs were found undergone major changes in organ-specific expression patterns in zebrafish and medaka, in comparison to gar $[9,56]$. Taken all these evidences together, it suggests neo- and sub-functionalization between Ohnologs is the reason for retention of both gene copies following genome duplication.

\section{Conclusions}

We conclude that despite structural duplication, functional redundancy of expression among gene duplicates is rare in most teleost organs. In addition, we provide an innovative collection of basal transcriptomic data from nine fish organs over a diurnal cycle, and provide a gene expression browser for research community to utilize.

\section{Methods}

\section{Fish utilized and RNA isolation}

$X$. maculatus Jp $163 \mathrm{~A}$ in the 116th inbred generation (i.e., sibling mating) were provided by the Xiphophorus Genetic Stock Center (http://www.xiphophorus.txstate. edu/). Mature (12 months old) male and female X. maculatus (12 months old) were maintained in separate 38-1 aquaria filled with filtered aquifer water from San Marcos, TX on a $13 \mathrm{~h} \mathrm{light} / 11 \mathrm{~h}$ dark cycle under $10,000 \mathrm{~K}$ fluorescent light (Coralife T8 Lamp 10,000 K, $32 \mathrm{~W}$ ). All fish utilized were co-adapted to the same conditions for at least 2 weeks prior to the experiment. Fish were fed with flake food precisely at $8 \mathrm{a} . \mathrm{m}$. and brine shrimp at $3 \mathrm{p} . \mathrm{m}$. during this adaptive synchronizing periods. A total of 24 male and 24 female Xiphophorus were used for this study. Two male fish were dissected for skin, brain, liver, heart, gills, muscle, eyes, and testis, two female fish were dissected for ovary at each Zt (Zt 0.5, 3.5, 6.5, 9.5, 12.5, 15.5, 18.5 and $21.5 \mathrm{~h}$; Supplement Figure 1). Heart samples were pooled from 3 males and 3 females at each time point to attain RNA yields required for construction of sequencing libraries. At dissection, fish were euthanized in an ice bath and upon loss of gill movement were sacrificed by cranial resection. All organs, except hearts, were dissected into separate $2.5 \mathrm{ml}$ microcentrifuge tubes filled with RNAlater (Ambion Inc.) for RNA isolation. A total of 48 animals were utilized ( 8 time point, 3 fish per sex per time point). All fish were maintained and samples taken in accordance with approved protocols (IACUC\# 2015107711).

Total RNA was isolated using a methodology similar to those previously detailed [57]. Briefly, RNAlater was removed from respective microcentrifuge tubes followed by addition of $750 \mu \mathrm{L}$ of QIAzol (Qiagen) in $2.0 \mathrm{~mL}$ collection tubes designed for automated tissue homogenization and RNA isolation stations. Organs were homogenized using the TisseLyser II (Qiagen) facilitated by stainless beads (Qiagen) for $10 \mathrm{~min}$ at $25 \mathrm{~Hz}$. RNA isolation was subsequently performed using a QIAcube HT (Qiagen) automated bio-sample isolation system. The isolation system is equipped with a robotic arm with 8 pipettes. Each pipette is able to pick and eject pipette tips, self-clean, and transfers liquids between well/columns, or between reagent reservoirs and well/columns in standard 96-well plate formats. Each sample was independently maintained throughout the isolation process. Briefly, $150 \mu \mathrm{L}$ of chloroform was added to each isolation tube and the samples were vigorously shaken for $15 \mathrm{~s}$ and then phases partitioned by centrifugation $\left(12,000 \times \mathrm{g}\right.$ for $15 \mathrm{~min}$ at $\left.4{ }^{\circ} \mathrm{C}\right)$. The aqueous phase containing nucleic acids was transferred to a new sample tube by a rack of automated pipettors. After extraction, nucleic acids were precipitated with $500 \mu \mathrm{L} 70 \%$ ethanol. RNA was then purified using a Qiagen RNeasy mini RNA kit (96-well plate) and eluted following the manufacturer's protocol. RNA was quantified with a Qubit 2.0 fluorometer (Life Technologies, Grand Island, NY, USA) and RNA quality was measured on an Agilent 2100 Bioanalyzer (Agilent Technologies, Santa Clara, CA) to confirm RIN scores were above 8.0 prior to sequencing. Concentrations of RNA samples were adjusted to $100 \mathrm{ng} / \mu \mathrm{L}$ with RNase-free water (Qiagen) prior to RNA sequencing.

\section{RNA-Seq and gene expression profiling}

Each RNA sample was used to construct an independent sequencing library using Illumina TruSeq library preparation kit (Illumina, Inc., San Diego, CA, USA). This library strategy led to a total of 136 dual-indexed libraries. Libraries were sequenced as $150 \mathrm{bp}$ paired-end fragments using Illumina HiSeq 2000 system (Illumina, Inc., San Diego, CA, USA). Sequencing files can be accessed in NCBI GEO with accession number GSE158968("https://www.ncbi. nlm.nih.gov/geo/query/acc.cgi?acc=GSE158968"). Short sequencing reads were filtered using an in-house data processing pipeline [58]. Briefly, sequencing adaptors, if detected, were first removed from sequencing reads. Processed sequencing reads were subsequently trimmed and filtered based on quality scores by using a filtration algorithm that removed low-scoring sections of each read and preserved the longest remaining fragment.

Processed sequencing reads were subsequently mapped to $X$. maculatus genome version 5.0 using Tophat2 [59], and gene expression of gene models annotated by Ensembl database (Release 94) was quantified using FeatureCount [60]. X. maculatus genome dataset, including genome sequence, genes, gene chromosomal coordinates are available at "https://uswest.ensembl.org/ Xiphophorus_maculatus/Info/Index".

\section{Detecting oscillating gene expression patterns}

Counts of sequencing reads were normalized to total read counts per sample to eliminate effects of different 
sequencing depths among the different samples. Normalized read counts were in the format of Count Per Million $(\mathrm{cpm})$ and were calculated as $\mathrm{cpm}=(\mathrm{read}$ counts $/$ library size) $\times 10^{6}$. Averaged $\mathrm{cpm}$ values were subsequently calculated using the biological replicates per time points. Relative expression at each time point was further calculated to bring all gene expression to the same scale: Relative expression $=($ Average $\mathrm{cpm}$ of biological replicate) / (Average cpm of all time points) [12].

Expressed genes of each organ were first identified by applying expression criteria to each gene of each organspecific expression profile. A gene is determined to be expressed if cpm values of at least two of the eight time points are larger than 1 . Subsequently, a Rhythmicity Analysis Incorporating Nonparametric algorithm was applied to identify genes exhibiting oscillating expression patterns [61]. The RAIN algorithm is a robust nonparametric method to detect rhythms. It first groups data by measurement time, then test the grouped data against oscillation model consisting of a rising and a falling slope (i.e., monatomic slopes). Slopes are independently tested by summation of Mann-Whitney-U-Tests. A $p$ value cutoff of 0.01 ( $p$-value $<0.01)$ was used to determine genes that showed oscillating expression patterns. Heatmaps, dot plots and line plots were used to represent the expression patterns of these genes over a single diurnal cycle. All plots were created using $\mathrm{R}$ plot function and heatmap.2 function of $\mathrm{R}$ package gplots.

\section{Syntenic group analyses of circadian master regulators}

Xiphophorus and gar chromosomal coordinates, and gar orthologs of Xiphophorus genes were downloaded from Ensembl database (Release 94). X. maculatus genome dataset, including genome sequence, genes, gene chromosomal coordinates are available at "https://uswest.ensembl. org/Xiphophorus_maculatus/Info/Index”. Gar genome dataset, including genome sequence, genes, gene chromosomal coordinates, are available at "https://uswest. ensembl.org/Lepisosteus_oculatus/Info/Index". Xiphophorus paralog gene IDs, paralog type, ancestry information, and gar orthologs of Xiphophorus genes, and transcript sequences are available at either "http://uswest. ensembl.org/biomart/martview/ae840c9e1d9e5837bec034 8203e881ab", or through R/Bioconductor Biomart at "https://www.bioconductor.org/packages/release/bioc/ html/biomaRt.html". For each Xiphophorus gene assigned to a location $i$ (gene ${ }_{i}$ ), its upstream (gene ${ }_{i+1} \ldots$ gene $_{i+n}$ ) and downstream neighbor genes $\left(\right.$ gene $_{i-n} \ldots$ gene $_{i-1}$ ) within a window $\mathrm{n}$ were acquired. Similarly, the gar ortholog of gene $i$ (ortholog ${ }_{i}$ ), along with its chromosome neighbors (ortholog $_{i+1} \ldots$ ortholog $_{i+n}$ and ortholog ${ }_{i-n} \ldots$ ortholog $_{i-1}$ ) were also retrieved using the gar annotation file. To test the "co-linearization" between the Xiphophorus gene and gar orthologs, each gene within the "gene ${ }_{i-n} \ldots$ gene $_{i+n}$ " were tested to each gar gene within the "ortholog $i-n \ldots$ ortholog $i+n$ " to test if Xiphophorus - gar orthology is present for each gene pair. A score is calculated as: Score = \# orthologs / (window size $\mathrm{n}$ ) $\times 2$. If a Xiphophorus gene appeared orthologous to several gar genes, the gene pair that results in the highest Score is assigned as the Xiphophorus - gar ortholog.

Circadian regulator gene families, which include any gene that contains "aryl hydrocarbon receptor nuclear translocator", "cryptochrome circadian", "period circadian", and "clock circadian", as well as paralogs of these genes' were retrieved from the Xiphophorus genome for synteny analyses compared to the gar.

\section{Identification of TGD Ohnologs in X. maculatus and spotted gar ortholog}

We employed the Pasquier et al. methodology (Pasquier et al., 2017) to identify X. maculatus Ohnolog pairs. Briefly, $X$. maculatus gene IDs ("ensembl_gene_id"), paralog IDs ("xmaculatus_paralog_ensembl_gene") and spotted gar ortholog gene IDs ("loculatus_homolog_ensembl_gene") were first downloaded from Ensembl Release 94 using Bioconductor package "Biomart". Paralog types and ancestry information ("xmaculatus_paralog_subtype" and "xmaculatus_ paralog_orthology_type", respectively) were also downloaded for paralogous pairs filter. To qualify as an Ohnolog pair, two $X$. maculatus paralogs are expected to be rooted from the TGD, and share one single spotted gar ortholog (i.e., 1:2 gene relationship between gar and $X$. maculatus). First, we removed any paralogous pairs that showed a duplication ancestor as "Clupeocephala", because this is a duplication point that occurs after the divergence of Xiphophorus from spotted gar. Second, paralogous pairs that exhibited more than one spotted gar ortholog were removed. Third, any gene that appeared more than once in all paralogous pairs was removed from the dataset. Additionally, we removed Ohnolog pairs that are not annotated to chromosomes (i.e., un-anchored contigs, and/or mitochondria), and are less than $5 \mathrm{Mbp}$ separated on the same chromosome. This process led to identification of 943 Ohnolog cases (i.e., 943 gar genes, 1886 Xiphophorus genes) where two Xiphophorus orthologs correspond to one gar gene.

\section{Identification of Xiphophorus singletons}

To identify Xiphophorus genes that show 1:1 gene relationship (i.e., singletons, or genes that lost one TGD Ohnolog in Xiphophorus) compared to the gar genes, we first retrieved ensembl gene ID of all Xiphophorus genes, their paralog gene IDs, paralog type and ancestry information, as well as gar orthologs of Xiphophorus genes from Ensembl Release 94 using Biomart. Next, paralogs that had an indication of TGD and post-TGD lineage specific duplication (i.e., "Clupeocephala", "Euteleosteomorpha", "Percomorphaceae", "Ovalentaria", "Atherinomorphae", 
"Cyprinodontlformes", "Cyprinodontoldel", "Poecllilnae", "Xiphophorus") were removed from the dataset. According to Pasquier et.al [9], genes with duplication ancestor "Neopterygii" because this duplication node can root from Ensembl tree reconstructions artifacts. Third, genes with gene names that suggest gene duplications (i.e., key work "one of many" in gene name) were removed. At last, each singleton is required to have one unique gar ortholog (i.e., L. oculatus orthology type $=$ "ortholog_one2one"). This analysis identified 10,952 Xiphophorus singletons compared the gar genome.

\section{Configuration of user interface and server}

The user interface (UI) depicting basal gene expression is designed using the $\mathrm{R}$ project Shiny platform (https:// cran.r-project.org/web/packages/shiny/index.html). To accurately deliver gene expression patterns, the Ensembl gene ID is required as the input due to ambiguity in common gene names that can confuse the algorithm. The Ensembl gene ID can be found using ensemble tool biomaRt, or the built-in gene ID table [62, 63].

Both UI ("ui" in Shiny is defined as the scripts that describe the user interface) and server ("server" in Shiny is defined as the scripts that process the data in correspondence to the "ui") were constructed using R language and were assembled as one single project. Once the user inputs a gene ID, the server collects basal expression data of 9 organs from the XGSC data server to calculate $\mathrm{cpm}$ and relative expression values resulting in two graphs, as well as a summarizing table: (a) A bar graph showing mean cpm values of a gene in all organs; (b) expression pattern of a gene throughout a 24-h period in all organs; (c) a table showing peak expression time of gene of interest, and if the gene(s) exhibit a circadian expression pattern (RAIN algorithm $p$-value $<0.01$ ). For flowchart, see Supplement Figure 2.

This project can be accessed remotely through the XGSC website (https://www.xiphophorus.txstate.edu), or downloaded from GitHub and launched locally using R command line. For detailed instructions, please see. https://www.xiphophorus.txstate.edu/Gene-Expression-Browser.html.

To utilize the user interface (UI), a query gene ID is required (Supplement Figures 3 and 4). The only input from the user is "Ensembl gene ID". This input format was selected because the gene ID (e.g., ENSXMAG00000016482) is unique to one single gene model, while the common gene name (e.g., egfr) in many cases has multiple gene entries in a given database. Additionally, it is estimated that $5-20 \%$ of the teleost genome remains duplicated from the Teleost Genome Duplication [9, 28, 64], rendering duplicate genes to share the same or similar gene names. Using ambiguous common gene names without an in-depth functional interpretation may lead to errors in experimental interpretation. For example, $X$. maculatus encodes two per2 genes resulting from the TGD. The loci that surround these two genes share orthology, and show synteny with the pre-TGD gar genome. They are annotated as "period circadian clock 2", and "period circadian regulator 2 ". Therefore, it is impossible to clearly distinguish the function of these two genes by these rather arbitrary names. The conversion of common gene name to gene ID can be accomplished using Ensemble Biomart, or by a built-in tool that searches for the Ensemble gene IDs for any gene that contains the user-input key word in a common gene name (Supplement Figure 3). After the gene ID is input into the "Ensembl Gene ID" window, the browser will download gene expression profiles of 9 organs (i.e., skin, brain, ovary, heart, muscle, eye, gill, liver and testis), collected every $3 \mathrm{~h}$ throughout a 24-h period and extract $\mathrm{cpm}$ values as gene expression values. The cpm values are next used to determine the expressiveness of a gene in different organs, and these data are plotted in a bar graph for visualization (Supplement Figure 4). Relative expression values for the gene-of-interest in each organ, at each time point, is also output as a plot to show basal expression level over the $24 \mathrm{~h}$ period (Supplement Figure 4). These two graphs are the main output of the presented browser that can assist users for study design. Along with these graphs is a summary table listing of the gene's expressivity in each organ, such as peak expression time, amplitude of expression changes over a day, and if it exhibits a circadian expression pattern (Supplement Figure 4).

\section{Comparison of circadian genes between Xiphophorus and zebrafish}

For a parallel comparison of the circadian genes between Xiphophorus and zebrafish, Ohnolog pairs, singleton genes and their gar orthologs were downloaded [9]. Known zebrafish circadian gene from brain and pineal gland, and their peak expression time were downloaded from circadian gene database [55] (http://cgdb.biocuckoo.org/). Uniprot IDs were first converted to Ensemble peptide IDs using Uniprot website user interface, and subsequently converted to Ensembl gene IDs using Biomart. Duplicated IDs due to different protein products encoded by the same gene were collapsed. If the same gene were identified to be circadian gene from several studies, only the study using RNA-Seq or microarray was kept. Circadian genes were separated to Ohnologs and singletons to assess sub-functionalization between Ohnologs in circadian expression pattern.

\section{Gene expression analysis by NanoString nCounter}

A panel of Nanostring nCounter capture and reporter probe set was custom designed to capture and quantify selected Xiphophorus transcripts [12]. This NanoString nCounter panel contained 4 Xiphophorus genes that exhibited cyclic expression patterns (Supplement Figure 10). 
Design and production of the nCounter probes, was performed by the Nanostring bioinformatics group (Nanostring, Seattle, WA). Transcript sequences corresponding to each gene target were downloaded from Ensembl database, and used as templates to design probes. Each probe is $100 \mathrm{nt}$ long, with a melting temperature between 73 and $91^{\circ} \mathrm{C}$ and will not form secondary structures inhibiting the assay. Probes were tested in silico to avoid cross hybridization to other loci. NanoString hybridization of RNA samples with the target panel was initiated by mixing $500 \mathrm{ng}$ of RNA $(100 \mathrm{ng} / \mu \mathrm{L})$ with the custom designed capture and reporter probe set. Samples were incubated for $12 \mathrm{~h}$ at $65^{\circ} \mathrm{C}$ and then processed by the NanoString Prep Station (NanoString Technologies, Seattle, WA, USA) and subsequent nCounter analysis to determine gene expression. Built-in positive control probes were used to control the binding efficiency of each sample as read counts generated by these probes are independent of the RNA samples. A scaling factor is calculated based on the mean value of positive control probe generated read counts. Samples with the scaling factor between 0.3 and 3 are qualified for further analyses. Subsequently, the scaled read counts were normalized to the geometrical mean of the housekeeping genes to normalize potentially different total RNA input. Finally, background signal noise was determined by the read counts of negative control probes and was removed from normalized read counts [65-67].

\section{Supplementary Information}

The online version contains supplementary material available at https://doi. org/10.1186/s12864-020-07202-9.

Additional file 1: Supplement Figure 1. Sample collection and experimental setup: 9 organs (skin, brain, liver, gill, heart, muscle, testis, ovary and eye) were collected at $5 \mathrm{am}, 8 \mathrm{am}, 11 \mathrm{am}, 2 \mathrm{pm}, 5 \mathrm{pm}, 8 \mathrm{pm}$, $11 \mathrm{pm}$ and $2 \mathrm{am}$. Duplicate samples of total RNA from each organ of individual fish was isolated for gene expression profiling. Supplement Figure 2. Flowchart depicting the configuration of the gene expression browser. Establishment of the gene expression browser includes 2 main steps: 1. Sample collection and gene expression profiling, and Gene ID and Gene name conversion: Gene expression data were normalized and scaled and stored in the XGSC website; All Xiphophorus Ensembl gene ID and corresponding gene name, as well as other bioinformatics information were downloaded into a custom table and stored in the XGSC website; 2. Configuration of the user interface and server: user interface was designed to convert common gene name to Ensembl gene ID to precisely identify the gene of interest, and to show basal gene expression pattern. These tools are stored in both shiny.io and Github for user to launch the browser remotely or locally. Supplement Figure 3. Illustration of the gene ID converter. The user interface (UI) contains a gene ID conversion tool. Users can input a common gene name as a key word to search through the whole genome to identify genes that may be of study interest. An example of "per" for period gene family is used in the Fig. 9 lines of data were returned. Among those two are gper1, a GPCR gene, and three are perp, a TP53 apoptosis effector gene that are all not relevant to the per gene family, and 4 per gene family members: per1b, per2, per3 and a per2 paralog (ENSMAG00000006651). Supplement Figure 4. Illustration of the gene expression browser user interface. Once in the web tool at the Xiphophorus Genetic Stock Center web page, an outside user can enter a proper Ensembl gene ID as input in order to generate a bar graph representing the organ-specific average expression levels of the query gene over a 24-h period. This generates an organspecific expression curve depicting the gene expression at each time point of a day. The example shown is per1b. The table underneath the bar graph and expression pattern plot shows basic statics of whether this gene exhibits a cyclic expression pattern, including gene name, description, peak time (in Zt), peak shape (i.e., hours to go from peak expression to the lowest expression level), amplitude (i.e., distance between peak expression and the bottom of a circadian expression pattern). Supplement Figure 5. Cyclic genes and opsin expression heatmaps representing (a) circadian genes distribution in each organ and (b) opsin expression in each organ. For each heatmap, each row represents a gene and each column represents an organ. Yellow blocks show a gene (a) exhibiting circadian expression pattern, or (b) expressed. Supplement Figure 6. Circadian master regulator gene orthology to gar. Three pairs of circadian master regulators in Xiphophorus were found to be Ohnologs. These genes include (a) arnt/2, (b) per2, (c) crylab. A syntenic block plot is used to show shared synteny of each Ohnolog with a gar ortholog. Red dots (open or closed) represent upstream and downstream neighboring genes of a Xiphophorus gene. The larger closed red dot represents a particular circadian gene. Blue dots (open or closed) represent upstream and downstream neighboring genes of gar ortholog of the Xiphophorus gene, and the larger closed blue dot represents the gar ortholog. Each Xiphophorus gene in the chromosome window is used to scan the gene of gar ortholog neighboring genes for orthology. If orthology exists, the Xiphophorus gene and the corresponding gar gene are connected by a gray line, with a closed dot representing Xiphophorus gene. Supplement Figure 7. Gene expression patterns of cyclic gene Ohnologs. A total of 626 Ohnologs pairs exhibited at least one of the duplicate showing circadian expression cycle in one organ. Per Ohnolog pair, 9 plots representing 9 organs were represent basal gene expression patterns, with solid line and dashed lines represented each gene duplicate. Supplement Figure 8. Gene expression patterns of singletons. A total of 4573 singletons exhibited circadian expression cycle in at least one organ. Per gene, 9 plots representing 9 organs were represent basal gene expression patterns, with solid line and dashed lines represented each gene duplicate. Supplement Figure 9. Expression analyses of cyclic gene Ohnologs exhibiting functional divergence in expression pattern: (A) intra-organ functional divergence, and (B) inter-organ functional divergence. In each case, two heatmap panels were used to describe (left panel) the expression status (i.e., gray = not expressed, black = expressed) and (right panel) relative expression between the Ohnologs (Ohnolog1 expression / Ohnolog2 expression). For the left panel, the center red line dividing each panel, splits the left and right half that represent Ohnolog1 and Ohnolog2, respectively. Status of expression and relative expression of Ohnologs are not correlated to if one Ohnolog exhibit circadian pattern, but not the other. $\mathrm{S}=$ Skin; $\mathrm{B}=$ Brain; $\mathrm{O}=$ Ovary; $\mathrm{H}=$ Heart; $\mathrm{M}=$ Muscle; $\mathrm{E}=$ Eye; $\mathrm{G}=\mathrm{Gill}$; $\mathrm{L}=$ Liver; $T=$ Testis. Supplement Figure 10. Validating expression patterns of 4 cyclic genes using nanostring nCounter. Expression patterns of 4 cyclic genes identified in X. maculatus skin using RNA-Seq (arnt/1, crylab, clockb, clocka) were assessed using nCounter by direct transcript molecule counting.

Additional file 2: Supplement Table 1. Differential expression analyses results.

Additional file 3: Supplement Table 2. Number of cyclic genes in each organ.

Additional file 4: Supplement Table 3. Cyclic genes of all 9 organs. Additional file 5: Supplement Table 4. Xiphophorus Ohnologs. Additional file 6: Supplement Table 5. Xiphophorus singletons. Additional file 7: Supplement Table 6. Number of cyclic Ohnologs and singletons.

Additional file 8: Supplement Table 7. Functional divergence of cyclic gene Ohnologs.

Additional file 9: Supplement Table 8. Circadian Ohnologs in zebrafish brain and pineal gland.

Additional file 10: Supplement Table 9. Genes showing circadian expression pattern in zebrafish brain and pineal gland. 


\section{Abbreviations}

Zt: Zeitgeber Time; WGD: whole genome duplication; TGD: Teleost genome duplication; CDS: Coding sequence; UTR: Untranslated region

\section{Acknowledgements}

The authors would like to thank the staff of the Xiphophorus Genetic Stock Center, Texas State University, for maintaining the pedigreed fish lines and caring for the animals used in this study.

\section{Authors' contributions}

All authors have read and approved the submitted version of the manuscript, and have agreed both to be personally accountable for the author's own contributions and to ensure that questions related to the accuracy or integrity of any part of the work, even ones in which the author was not personally involved, are appropriately investigated, resolved, and the resolution documented in the literature. Authors' contribution are, 1. Study design: YL, MB, WB, MS1, RW; 2. acquisition, analysis of data: $Y L, M B, W B, R S$, MS1, MS2, JR, SW, RM, TG, GM, WW, RW; interpretation of data: YL, MB, WB, $R S, W W, M S 2, R B$; creation of new software used in the work: $Y L, W B$; manuscript drafting and revising: YL, MB, WB, RS, WW, MS2, RB; Acquisition of funds: YL, WW, MS2, RW.

\section{Funding}

The National Institute of Health $(\mathrm{NIH})$ provided support for this project. Funds include NIH-Office of Research Infrastructure Program (ORIP) grants R24-OD-011120, R24OD-018555, NIH-National Cancer Institute (NCI) grant R15-CA-223964 and NIH-National Institute of General Medical Sciences (NIGM S) grant R25-GM-102783-06. The funders had no role in study design, data collection and analysis, decision to publish, or preparation of the manuscript.

\section{Availability of data and materials}

$X$. maculatus genome dataset, including genome sequence, genes, gene chromosomal coordinates are available at "https://uswest.ensembl.org/ Xiphophorus_maculatus/Info/Index". This dataset is associated to Supplement Tables 1, 3, 4, 5, and 7. Gar genome dataset, including genome sequence, genes, gene chromosomal coordinates, are available at "https:// uswest.ensembl.org/Lepisosteus_oculatus/Info/Index". The gar genome dataset is associated to Supplement Tables 4, 5, and 7. Xiphophorus paralog gene IDs, paralog type, ancestry information, and gar orthologs of Xiphophorus genes, and transcript sequences are available at either "http:// uswest.ensembl.org/biomart/martview/ae840c9e1d9e5837bec0348203e881 ab", or through R/Bioconductor Biomart at "https://www.bioconductor.org/ packages/release/bioc/html/biomaRt.html". This information is related to Supplement Tables 4, 5, and 7. Sequencing files can be accessed in NCBI GEO with accession number GSE158968 ("https://www.ncbi.nlm.nih.gov/geo/ query/acc.cgi?acc=GSE158968"). Zebrafish circadian gene sets were downloaded from circadian gene database at "http://cgdb.biocuckoo.org/", and are associated to Supplement Tables 8 and 9.

\section{Ethics approval and consent to participate}

All fish were kept and samples taken in accordance with protocols approved by Texas State University IACUC (IACUC2015107711).

\section{Consent for publication}

Not applicable.

\section{Competing interests}

The authors declare no competing interest.

\section{Author details}

${ }^{1}$ The Xiphophorus Genetic Stock Center, Department of Chemistry and Biochemistry, Texas State University, 419 Centennial Hall, 601 University Drive, San Marcos, TX 78666, USA. ${ }^{2}$ The University of Texas MD Anderson Cancer Center, Graduate School of Biomedical Sciences, Houston, TX, USA. ${ }^{3}$ Bond Life Sciences Center, University of Missouri, Columbia, MO, USA ${ }^{4}$ Developmental Biochemistry, Theodor-Boveri-Institute, Biozentrum, University of Würzburg, Würzburg, Germany.
Received: 27 January 2020 Accepted: 28 October 2020

Published online: 11 November 2020

\section{References}

1. Nelson J. Fishes of the world. 3rd ed. Hoboken: Wiley; 1994

2. Volff JN. Genome evolution and biodiversity in teleost fish. Heredity. 2005; 94(3):280-94.

3. Santini F, Harmon LJ, Carnevale G, Alfaro ME. Did genome duplication drive the origin of teleosts? A comparative study of diversification in ray-finned fishes. BMC Evol Biol. 2009;9:194.

4. Glasauer SM, Neuhauss SC. Whole-genome duplication in teleost fishes and its evolutionary consequences. Mol Gen Genomics. 2014;289(6):1045-60.

5. Force A, Lynch M, Pickett FB, Amores A, Yan YL, Postlethwait J. Preservation of duplicate genes by complementary, degenerative mutations. Genetics. 1999:151(4):1531-45.

6. Yu WP, Brenner S, Venkatesh B. Duplication, degeneration and subfunctionalization of the nested synapsin-Timp genes in Fugu. Trends Genet. 2003;19(4):180-3.

7. He X, Zhang J. Rapid subfunctionalization accompanied by prolonged and substantial neofunctionalization in duplicate gene evolution. Genetics. 2005; 169(2):1157-64.

8. Inoue J, Sato $Y$, Sinclair R, Tsukamoto K, Nishida M. Rapid genome reshaping by multiple-gene loss after whole-genome duplication in teleost fish suggested by mathematical modeling. Proc Natl Acad Sci U S A. 2015; 112(48):14918-23.

9. Pasquier J, Braasch I, Batzel P, Cabau C, Montfort J, Nguyen T, Jouanno E, Berthelot C, Klopp C, Journot L, et al. Evolution of gene expression after whole-genome duplication: new insights from the spotted gar genome. J Exp Zool B Mol Dev Evol. 2017;328(7):709-21.

10. Giglia-Mari G, Theil AF, Mari PO, Mourgues S, Nonnekens J, Andrieux LO, de Wit J, Miquel C, Wijgers N, Maas A, et al. Differentiation driven changes in the dynamic organization of basal transcription initiation. PLoS Biol. 2009; 7(10):e1000220

11. Yosef $\mathrm{N}$, Regev A. Impulse control: temporal dynamics in gene transcription. Cell. 2011;144(6):886-96

12. Lu Y, Reyes J, Walter S, Gonzalez T, Medrano G, Boswell M, Boswell W, Savage M, Walter R. Characterization of basal gene expression trends over a diurnal cycle in Xiphophorus maculatus skin, brain and liver. Comp Biochem Physiol Toxicol Pharmacol. 2018;208:2-11.

13. Merrow M, Spoelstra K, Roenneberg T. The circadian cycle: daily rhythms from behaviour to genes. EMBO Rep. 2005;6(10):930-5.

14. Kaneko M, Cahill GM. Light-dependent development of circadian gene expression in transgenic zebrafish. PLoS Biol. 2005;3(2):e34.

15. Jang H, Lee G, Kong J, Choi G, Park YJ, Kim JB. Feeding period restriction alters the expression of peripheral circadian rhythm genes without changing body weight in mice. PLoS One. 2012;7(11):e49993.

16. Atger F, Gobet C, Marquis J, Martin E, Wang J, Weger B, Lefebvre G, Descombes P, Naef F, Gachon F. Circadian and feeding rhythms differentially affect rhythmic mRNA transcription and translation in mouse liver. Proc Natl Acad Sci U S A. 2015;112(47):E6579-88.

17. Boswell M, Boswell W, Lu Y, Savage M, Mazurek Z, Chang J, Muster J, Walter $R$. The transcriptional response of skin to fluorescent light exposure in viviparous (Xiphophorus) and oviparous (Danio, Oryzias) fishes. Comp Biochem Physiol Toxicol Pharmacol. 2018;208:77-86.

18. Gonzalez TJ, Lu Y, Boswell M, Boswell W, Medrano G, Walter S, Ellis S, Savage M, Varga ZM, Lawrence $C$, et al. Fluorescent light exposure incites acute and prolonged immune responses in zebrafish (Danio rerio) skin. Comp Biochem Physiol Toxicol Pharmacol. 2018;208:87-95.

19. Walter RB, Boswell M, Chang J, Boswell WT, Lu Y, Navarro K, Walter SM, Walter DJ, Salinas R, Savage M. Waveband specific transcriptional control of select genetic pathways in vertebrate skin (Xiphophorus maculatus). BMC Genomics. 2018;19(1):355.

20. Panda S, Hogenesch JB, Kay SA. Circadian rhythms from flies to human. Nature. 2002:417(6886):329-35.

21. Bell-Pedersen D, Cassone VM, Earnest DJ, Golden SS, Hardin PE, Thomas TL, Zoran MJ. Circadian rhythms from multiple oscillators: lessons from diverse organisms. Nat Rev Genet. 2005;6(7):544-56

22. Foster R, Kreitzman L. Rhythms of life: the biological clocks that control the daily lives of every living thing. New Haven: Yale University Press; 2005.

23. Takahashi JS. Transcriptional architecture of the mammalian circadian clock. Nat Rev Genet. 2017;18(3):164-79. 
24. Beale A, Guibal C, Tamai TK, Klotz L, Cowen S, Peyric E, Reynoso VH, Yamamoto Y, Whitmore D. Circadian rhythms in Mexican blind cavefish Astyanax mexicanus in the lab and in the field. Nat Commun. 2013;4:2769.

25. Kim BM, Amores A, Kang S, Ahn DH, Kim JH, Kim IC, Lee JH, Lee SG, Lee H, Lee J, et al. Antarctic blackfin icefish genome reveals adaptations to extreme environments. Nat Ecol Evol. 2019;3(3):469-78.

26. Toloza-Villalobos J, Arroyo JI, Opazo JC. The circadian clock of teleost fish: a comparative analysis reveals distinct fates for duplicated genes. J Mol Evol. 2015;80(1):57-64.

27. Froland Steindal IA, Whitmore D. Circadian clocks in fish-what have we learned so far? Biology. 2019;8(1).

28. Jaillon O, Aury JM, Brunet F, Petit JL, Stange-Thomann N, Mauceli E, Bouneau L, Fischer C, Ozouf-Costaz C, Bernot A, et al. Genome duplication in the teleost fish Tetraodon nigroviridis reveals the early vertebrate protokaryotype. Nature. 2004;431(7011):946-57.

29. Ravi V, Venkatesh B. The divergent genomes of Teleosts. Ann Rev Anim Biosci. 2018;6:47-68.

30. Schartl M. Beyond the zebrafish: diverse fish species for modeling human disease. Dis Model Mech. 2014;7(2):181-92.

31. Schartl M, Walter RB, Shen Y, Garcia T, Catchen J, Amores A, Braasch I, Chalopin D, Volff JN, Lesch KP, et al. The genome of the platyfish, Xiphophorus maculatus, provides insights into evolutionary adaptation and several complex traits. Nat Genet. 2013;45(5):567-72.

32. Shen Y, Chalopin D, Garcia T, Boswell M, Boswell W, Shiryev SA, Agarwala R, Volff JN, Postlethwait JH, Schartl M, et al. X couchianus and X hellerii genome models provide genomic variation insight among Xiphophorus species. BMC Genomics. 2016;17:37.

33. Lu Y, Boswell M, Boswell W, Kneitz S, Hausmann M, Klotz B, Regneri J, Savage M, Amores A, Postlethwait J, et al. Molecular genetic analysis of the melanoma regulatory locus in Xiphophorus interspecies hybrids. Mol Carcinog. 2017;56(8):1935-44.

34. Lu Y, Zhang C, Li Q, Mao J, Ma W, Yu X, Hou Z, Li L: [lnhibitory effect of salinomycin on human breast cancer cells MDA-MB-231 proliferation through hedgehog signaling pathway]. Zhonghua Bing Li Xue Za Zhi 2015, 44(6):395-398.

35. Lu Y, Bowswell M, Bowswell W, Yang K, Schartl M, Walter RB. Molecular genetic response of Xiphophorus maculatus-X. couchianus interspecies hybrid skin to UVB exposure. Comp Biochem Physiol Toxicol Pharmacol. 2015;178:86-92.

36. Liu C, Hu J, Qu C, Wang L, Huang G, Niu P, Zhong Z, Hong F, Wang G, Postlethwait JH, et al. Molecular evolution and functional divergence of zebrafish (Danio rerio) cryptochrome genes. Sci Rep. 2015;5:8113.

37. Lin C, Todo T. The cryptochromes. Genome Biol. 2005;6(5):220.

38. Lucas-Lledo Jl, Lynch M. Evolution of mutation rates: phylogenomic analysis of the photolyase/cryptochrome family. Mol Biol Evol. 2009;26(5):1143-53.

39. Mei Q, Dvornyk V. Evolutionary history of the Photolyase/Cryptochrome superfamily in eukaryotes. PLoS One. 2015;10(9):e0135940.

40. Walter RB, Walter DJ, Boswell WT, Caballero KL, Boswell M, Lu Y, Chang J, Savage MG. Exposure to fluorescent light triggers down regulation of genes involved with mitotic progression in Xiphophorus skin. Comp Biochem Physiol Toxicol Pharmacol. 2015;178:93-103.

41. Walter DJ, Boswell M, Volk de Garcia SM, Walter SM, Breitenfeldt EW, Boswell W, Walter RB. Characterization and differential expression of CPD and 6-4 DNA photolyases in Xiphophorus species and interspecies hybrids. Comp Biochem Physiol Toxicol Pharmacol. 2014;163:77-85.

42. Alvarez JD, Chen D, Storer E, Sehgal A. Non-cyclic and developmental stage-specific expression of circadian clock proteins during murine spermatogenesis. Biol Reprod. 2003;69(1):81-91.

43. Morse D, Cermakian N, Brancorsini S, Parvinen M, Sassone-Corsi P. No circadian rhythms in testis: period 1 expression is clock independent and developmentally regulated in the mouse. Mol Endocrinol. 2003;17(1):141-51.

44. Fahrenkrug J, Georg B, Hannibal J, Hindersson P, Gras S. Diurnal rhythmicity of the clock genes Per1 and Per2 in the rat ovary. Endocrinology. 2006; 147(8):3769-76.

45. Bebas P, Goodall CP, Majewska M, Neumann A, Giebultowicz JM, Chappell PE. Circadian clock and output genes are rhythmically expressed in extratesticular ducts and accessory organs of mice. FASEB J. 2009;23(2):523-33.

46. Chen H, Gao L, Xiong Y, Yang D, Li C, Wang A, Jin Y. Circadian clock and steroidogenic-related gene expression profiles in mouse Leydig cells following dexamethasone stimulation. Biochem Biophys Res Commun. 2017;483(1):294-300.
47. Shimizu T, Watanabe K, Anayama N, Miyazaki K. Effect of lipopolysaccharide on circadian clock genes Per2 and Bmal1 in mouse ovary. J Physiol Sci. 2017;67(5):623-8.

48. Khan ZA, Labala RK, Yumnamcha T, Devi SD, Mondal G, Sanjita Devi H, Rajiv C, Bharali R, Chattoraj A. Artificial light at night (ALAN), an alarm to ovarian physiology: a study of possible chronodisruption on zebrafish (Danio rerio). Sci Total Environ. 2018;628-629:1407-21.

49. Amores A, Catchen J, Ferrara A, Fontenot Q, Postlethwait JH. Genome evolution and meiotic maps by massively parallel DNA sequencing: spotted gar, an outgroup for the teleost genome duplication. Genetics. 2011;188(4): 799-808.

50. Braasch I, Gehrke AR, Smith JJ, Kawasaki K, Manousaki T, Pasquier J, Amores A, Desvignes T, Batzel P, Catchen J, et al. The spotted gar genome illuminates vertebrate evolution and facilitates human-teleost comparisons. Nat Genet. 2016:48(4):427-37.

51. Guo B. Complex genes are preferentially retained after whole-genome duplication in teleost fish. J Mol Evol. 2017;84(5-6):253-8.

52. Vatine $G$, Vallone D, Gothilf $Y$, Foulkes NS. It's time to swim! Zebrafish and the circadian clock. FEBS Lett. 2011;585(10):1485-94.

53. Foulkes NS, Whitmore D, Vallone D, Bertolucci C. Studying the evolution of the vertebrate circadian clock: the power of fish as comparative models. Adv Genet. 2016:95:1-30.

54. Lynch M, Conery JS. The evolutionary fate and consequences of duplicate genes. Science. 2000;290(5494):1151-5.

55. Li S, Shui K, Zhang Y, LV Y, Deng W, Ullah S, Zhang L, Xue Y. CGDB: a database of circadian genes in eukaryotes. Nucleic Acids Res. 2017;45(D1): D397-403.

56. Pasquier J, Cabau C, Nguyen T, Jouanno E, Severac D, Braasch I, Journot L, Pontarotti P, Klopp C, Postlethwait JH, et al. Gene evolution and gene expression after whole genome duplication in fish: the PhyloFish database. BMC Genomics. 2016;17:368.

57. Lu Y, Boswell W, Boswell M, Klotz B, Kneitz S, Regneri J, Savage M, Mendoza C, Postlethwait J, Warren WC, et al. Application of the transcriptional disease signature (TDSs) to screen melanoma-effective compounds in a small fish model. Sci Rep. 2019;9(1):530.

58. Garcia TI, Shen Y, Catchen J, Amores A, Schartl M, Postlethwait J, Walter RB. Effects of short read quality and quantity on a de novo vertebrate transcriptome assembly. Comp Biochem Physiol Toxicol Pharmacol. 2012; 155(1):95-101.

59. Kim D, Pertea G, Trapnell C, Pimentel H, Kelley R, Salzberg SL. TopHat2: accurate alignment of transcriptomes in the presence of insertions, deletions and gene fusions. Genome Biol. 2013;14(4):R36.

60. Liao Y, Smyth GK, Shi W. The subread aligner: fast, accurate and scalable read mapping by seed-and-vote. Nucleic Acids Res. 2013;41(10):e108.

61. Thaben PF, Westermark PO. Detecting rhythms in time series with RAIN. J Biol Rhythm. 2014;29(6):391-400.

62. Durinck S, Spellman PT, Birney E, Huber W. Mapping identifiers for the integration of genomic datasets with the R/Bioconductor package biomaRt. Nat Protoc. 2009;4(8):1184-91.

63. Durinck S, Moreau Y, Kasprzyk A, Davis S, De Moor B, Brazma A, Huber W. BioMart and bioconductor: a powerful link between biological databases and microarray data analysis. Bioinformatics. 2005;21(16):3439-40.

64. Christoffels A, Koh EG, Chia JM, Brenner S, Aparicio S, Venkatesh B. Fugu genome analysis provides evidence for a whole-genome duplication early during the evolution of ray-finned fishes. Mol Biol Evol. 2004;21(6):1146-51.

65. Brumbaugh CD, Kim HJ, Giovacchini M, Pourmand N. NanoStriDE: normalization and differential expression analysis of NanoString nCounter data. BMC Bioinformatics. 2011;12:479.

66. Malkov VA, Serikawa KA, Balantac N, Watters J, Geiss G, Mashadi-Hossein A, Fare T. Multiplexed measurements of gene signatures in different analytes using the Nanostring nCounter assay system. BMC Res Notes. 2009;2:80.

67. Geiss GK, Bumgarner RE, Birditt B, Dahl T, Dowidar N, Dunaway DL, Fell HP, Ferree S, George RD, Grogan T, et al. Direct multiplexed measurement of gene expression with color-coded probe pairs. Nat Biotechnol. 2008;26(3): $317-25$.

\section{Publisher's Note}

Springer Nature remains neutral with regard to jurisdictional claims in published maps and institutional affiliations. 